\title{
Exponential stability analysis for delayed stochastic Cohen-Grossberg neural network
}

\author{
Guanjun Wang, Jinling Liang \\ Department of Mathematics, Southeast University, Nanjing 210096, China \\ E-mail:wgjmath@gmail.com,jinlliang@gmail.com \\ Received: 24-11-2008 \\ Accepted: 01-12-2009
}

\begin{abstract}
In this paper, the exponential stability problems are addressed for a class of delayed Cohen-Grossberg neural networks which are also perturbed by some stochastic noises. By employing the Lyapunov method, stochastic analysis and some inequality techniques, sufficient conditions are acquired for checking the $p t h(p>1)$ and the $1 s t$ moment exponential stability of the network. Finally, One example is given to show the effectiveness of the proposed results.
\end{abstract}

Keywords: Exponential stability, Cohen-Grossberg neural networks, Lyapunov method, Razumikhin-type theorem, Brownian motion, Itô's formula.

\section{Introduction}

The Cohen-Grossberg neural network (CGNN) model was introduced by Cohen and Grossberg in $1983^{1}$ and has been extensively studied by many scholars for its important applications such as pattern recognition, associative memory, etc. Much work has been done to investigate the stability, boundedness and other dynamical behaviors of the networks ${ }^{2,3,4}$, which are helpful for the design of the neural networks. In the literature, most research focuses on the deterministic model. However, it should be pointed out that neural networks often work in some kinds of noise circumstance which may bring stochastic disturbance to the inputs of the networks. Results in ${ }^{5,6}$ suggested that the neural networks can be stabilized or destabilized by certain stochastic inputs, which implies that it is important to consider the noise effects in the stability analysis for the neural networks. Recently, the study of stochastic neural networks has drawn much atten- tions from researchers all over the world and some results can be found in 7,8,9,10,11,12,13,22 and the references cited therein $15,16,17,19,21$. But for the study of stochastic CGNN, up till now, there are only a few results $10,11,14,20$. For example, Zhao ${ }^{10}$ discussed the almost sure exponential stability by using the semimartingale convergence theorem, and Wang et al ${ }^{11}$ obtained several asymptotic stability criteria by applying the well-known Lyapunov functional approach ${ }^{18}$.

In this paper, the stochastic CGNN will be studied in a different way comparing to Zhao ${ }^{10}$ or Wang 11. Firstly, the stochastic version of Razumikhintype theorem constructed by Mao ${ }^{12}$ is utilized to give some sufficient conditions ensuring the $p \operatorname{th}(p>$ 1) moment exponential stability of the networks. Then, by employing a suitable Lyapunov function and some analysis techniques, a sufficient condition is derived for the $1 s t$ moment exponential stability of the CGNN. In the end of this paper, an example is demonstrated to illustrate the proposed criteria and a 
comparison with the criteria introduced in ${ }^{11}$ is also provided.

Notations: Throughout this paper, $R^{+}$and $R_{+}$ represent, respectively, the set of all positive real numbers and the set of all nonnegative real numbers. $R^{n}$ is the $n$-dimensional Euclidean space. Let $\tau>0$ and $C\left([-\tau, 0] ; R^{n}\right)$ denotes the family of continuous functions $\varphi(\cdot)$ from $[-\tau, 0]$ to $R^{n}$ with the norm defined by $\|\varphi\|=\sup _{\tau \leqslant \theta \leqslant 0}|\varphi(\theta)|$, where $|\cdot|$ is the Euclidean norm in $R^{n} .|x(t)|_{1}=$ $\sum_{i=1}^{n}\left|x_{i}(t)\right|$ represents the 1-norm of vector $x(t) \in R^{n}$ and $w(t)=\left(w_{1}(t), w_{2}(t), \ldots, w_{n}(t)\right)^{T}$ means an $n$ dimensional Brownian motion defined on a complete probability space $(\Omega, \mathscr{F}, \mathscr{P})$ with a natural filtration $\left\{\mathscr{F}_{t}\right\}_{t \geqslant 0} . C_{\mathscr{F}_{0}}^{b}\left([-\tau, 0] ; R^{n}\right)$ means the family of all bounded, $\mathscr{F}_{0}$-measurable, $C\left([-\tau, 0] ; R^{n}\right)$ valued random variables. For $p \geqslant 1$ and $t \geqslant$ 0 , denote by $L_{\mathscr{F}_{t}}^{p}\left([-\tau, 0] ; R^{n}\right)$ the family of all bounded, $\mathscr{F}_{t}$-measurable $C\left([-\tau, 0] ; R^{n}\right)$-valued random variables $\varphi=\{\varphi(\theta):-\tau \leqslant \theta \leqslant 0\}$ such that $\sup _{-\tau \leqslant \theta \leqslant 0} E|\varphi(\theta)|^{p}<\infty$.

\section{Model Formulation and Preliminaries}

Consider the following delayed stochastic CGNN:

$$
\left\{\begin{aligned}
d x_{i}(t)= & -d_{i}\left(x_{i}(t)\right)\left[c_{i}\left(x_{i}(t)\right)-\sum_{j=1}^{n} a_{i j} f_{j}\left(x_{j}(t)\right)\right. \\
& \left.-\sum_{j=1}^{n} b_{i j} f_{j}\left(x_{j}(t-\tau)\right)\right] d t \\
& +\sum_{j=1}^{n} \sigma_{i j}\left(x_{j}(t), x_{j}(t-\tau)\right) d w_{j}(t), \\
x_{i}(t)= & \xi_{i}(t), \quad-\tau \leqslant t \leqslant 0 .
\end{aligned}\right.
$$

Throughout this paper, the following assumptions are made:

(H1) There exist positive constants $m_{i}$ and $M_{i}$ such that

$$
0<m_{i} \leqslant d_{i}(\cdot) \leqslant M_{i}, \quad i=1,2, \ldots, n .
$$

$(H 2) c_{i}(\cdot)$ is differentiable and

$$
\alpha_{i}=\inf _{x \in R} c_{i}^{\prime}(x)>0, \quad c_{i}(0)=0, \quad i=1,2, \ldots, n .
$$

(H3) The nonlinear functions $f_{i}(\cdot)(i=1,2, \ldots, n)$ are globally Lipschitz continuous and $f_{i}(0)=0$, i.e. there exist positive scalars $\beta_{i}$ such that

$$
\left|f_{i}(x)-f_{i}(y)\right|<\beta_{i}|x-y|, \quad \forall x, y \in R .
$$

(H4) The nonlinear functions $\sigma_{i j}(\cdot) \quad(i, j=$ $1,2, \ldots, n)$ is globally Lipschitz continuous and $\sigma_{i, j}(0,0)=0$, i.e. there exist positive constants $l_{i j}$ and $k_{i j}$ such that

$\left|\sigma_{i j}\left(x_{2}, y_{2}\right)-\sigma_{i j}\left(x_{1}, y_{1}\right)\right| \leqslant l_{i j}\left|x_{2}-x_{1}\right|+k_{i j}\left|y_{2}-y_{1}\right|$

holds for all $x_{1}, x_{2}, y_{1}, y_{2} \in R$.

To prove our main results, we need the following lemmas and notations:

Lemma 1. [Young Inequality] For any $x, y, p, q \in$ $R^{+}$with $1 / p+1 / q=1$, one has

$$
x y \leqslant \frac{1}{p} x^{p}+\frac{1}{q} y^{q} .
$$

Consider a stochastic functional differential equation

$$
\left\{\begin{aligned}
d x(t) & =f\left(x_{\tau}, t\right) d t+g\left(x_{\tau}, t\right) d w(t), \\
x(t) & =\xi(t) \in R^{n}, \quad-\tau \leqslant t \leqslant 0 .
\end{aligned}\right.
$$

Let $C^{1,2}\left(R^{n} \times[-\tau, \infty) ; R_{+}\right)$be the family of all nonnegative functions which are continuously once differentiable in $t$ and twice differentiable in $x$; for $V \in C^{1,2}\left(R^{n} \times[-\tau, \infty) ; R_{+}\right)$, define the operator $\mathscr{L} V$ for system (2) by

$$
\begin{array}{r}
\mathscr{L} V(\phi, t)=V_{t}(\phi(0), t)+V_{x}(\phi(0), t) f(\phi, t) \\
+\frac{1}{2} \operatorname{trace}\left[g^{T}(\phi, t) V_{x x}(\phi(0), t) g(\phi, t)\right],
\end{array}
$$

where

$$
\begin{aligned}
V_{t}(x, t) & =\frac{\partial V(x, t)}{\partial t}, \\
V_{x x}(x, t) & =\left(\frac{\partial^{2} V(x, t)}{\left(\partial x_{i} \partial x_{j}\right)}\right)_{n \times n}, \\
V_{x}(x, t) & =\left(\frac{\partial V(x, t)}{\partial x_{1}}, \frac{\partial V(x, t)}{\partial x_{2}}, \ldots, \frac{\partial V(x, t)}{\partial x_{n}}\right) .
\end{aligned}
$$

Lemma 2. (Razumikhin-type Theorem ${ }^{5}$ ) For system (2), assume that $f$, g satisfy the Lipschitz condition and the linear growth condition. Let $\lambda, p, c_{1}, c_{2}$ be all positive numbers and $q>1$. Assume that there 
exists a function $V(x, t) \in C^{1,2}\left(R^{n} \times[-\tau, \infty) ; R_{+}\right)$ such that

$c_{1}|x|^{p} \leqslant V(x, t) \leqslant c_{2}|x|^{p}, \quad \forall(x, t) \in R^{n} \times[-\tau, \infty) ;$

and also for all $t \geqslant 0$,

$$
E \mathscr{L} V(\phi, t) \leqslant-\lambda E V(\phi(0), t)
$$

provided $\phi=\{\phi(\theta):-\tau \leqslant \theta \leqslant 0\} \in$ $L_{\mathscr{F}_{t}}^{p}\left([-\tau, 0] ; R^{n}\right)$ satisfying

$E V(\phi(\theta), t+\theta) \leqslant q E V(\phi(0), t), \forall-\tau \leqslant \theta \leqslant 0$.

Then for all $\xi \in C_{\mathscr{F}_{0}}^{b}\left([-\tau, 0] ; R^{n}\right)$,

$$
E|x(\xi, t)|^{p} \leqslant \frac{c_{2}}{c_{1}} E\|\xi\|^{p} e^{-\gamma t}, \quad \forall t \geqslant 0
$$

where $\gamma=\min \{\lambda, \log (q) / \tau\}$.

Definition 1. ${ }^{12}$ The trivial solution of system (1) is said to be pth moment exponentially stable if there exists a pair of positive constants $\lambda$ and $C$ such that

$$
E|x(\xi, t)|^{p} \leqslant C E\|\xi\|^{p} e^{-\lambda t}, \quad t \geqslant 0
$$

holds for any $\xi \in L_{\mathscr{F}_{t}}^{p}\left([-\tau, 0] ; R^{n}\right)$. Especially, when $p=2$, it is usually called to be exponentially stable in mean square. When $p=1$, it is called to be $1 s t$ moment exponentially stable.

\section{Main Results}

In this section, some stability criteria are obtained for the delayed stochastic CGNN (1).

Theorem 3. Let Assumptions (H1) - (H4) hold. For constant $p \geqslant 2$, system (1) is pth moment exponentially stable if $\lambda_{1}>\lambda_{2}$, where

$$
\begin{aligned}
\lambda_{1}= & \min _{1 \leqslant i \leqslant n}\left\{p \alpha_{i} m_{i}-(p-1) M_{i}\left(\sum_{j=1}^{n}\left(\left|a_{i j}\right|+\left|b_{i j}\right|\right) \beta_{j}\right)\right. \\
& -(p-1)(p-2) \sum_{j=1}^{n}\left(l_{i j}^{2}+k_{i j}^{2}\right) \\
& \left.-\beta_{i}\left(\sum_{j=1}^{n}\left|a_{j i}\right| M_{j}\right)-2(p-1) \sum_{j=1}^{n} l_{j i}^{2}\right\} \\
\lambda_{2}= & \max _{1 \leqslant i \leqslant n}\left\{\beta_{i}\left(\sum_{j=1}^{n}\left|b_{j i}\right| M_{j}\right)+2(p-1) \sum_{j=1}^{n} k_{j i}^{2}\right\}
\end{aligned}
$$

Proof. Define a Lyapunov function $V(x, t)$ as follows:

$$
V(x, t)=\sum_{i=1}^{n}\left|x_{i}(t)\right|^{p} .
$$

Denote $\Sigma=\operatorname{diag}\left\{\left|x_{1}(t)\right|^{p-2},\left|x_{2}(t)\right|^{p-2}, \ldots,\left|x_{n}(t)\right|^{p-2}\right\}$ and $\sigma(x(t), x(t-\tau))=\left(\sigma_{i j}\left(x_{j}(t), x_{j}(t-\tau)\right)\right)_{n \times n}$, from formula (3) and Lemma 1 one can obtain

$$
\begin{aligned}
& \mathscr{L} V(x, t) \\
= & \sum_{i=1}^{n} p\left|x_{i}(t)\right|^{p-1} \operatorname{sign}\left(x_{i}(t)\right)\left\{-d_{i}\left(x_{i}(t)\right)\left[c_{i}\left(x_{i}(t)\right)\right.\right. \\
& \left.\left.-\sum_{j=1}^{n} a_{i j} f_{j}\left(x_{j}(t)\right)-\sum_{j=1}^{n} b_{i j} \times f_{j}\left(x_{j}(t-\tau)\right)\right]\right\} \\
& +\frac{p(p-1)}{2} \operatorname{trace}\left[\sigma^{T}\left(x(t), x_{\tau}(t)\right) \Sigma \sigma\left(x(t), x_{\tau}(t)\right)\right] \\
\leqslant & -p \sum_{i=1}^{n} \alpha_{i} m_{i}\left|x_{i}(t)\right|^{p}+p \sum_{i=1}^{n} M_{i}\left|x_{i}(t)\right|^{p-1} \\
& \times\left[\sum_{j=1}^{n}\left|a_{i j}\right| \beta_{j}\left|x_{j}(t)\right|+\sum_{j=1}^{n}\left|b_{i j}\right| \beta_{j} \times\left|x_{j}(t-\tau)\right|\right] \\
& +p(p-1) \sum_{i=1}^{n} \sum_{j=1}^{n}\left|x_{i}(t)\right|^{p-2}\left[l_{i j}^{2}\left|x_{j}(t)\right|^{2}\right. \\
& \left.+k_{i j}^{2}\left|x_{j}(t-\tau)\right|^{2}\right] \\
\leqslant & -p \sum_{i=1}^{n} \alpha_{i} m_{i}\left|x_{i}(t)\right|^{p} \\
& +(p-1) \sum_{i=1}^{n} M_{i}\left(\sum_{j=1}^{n}\left|a_{i j}\right| \beta_{j}\right)\left|x_{i}(t)\right|^{p} \\
& +2(p-1) \sum_{i=1}^{n} \sum_{j=1}^{n} l_{j i}^{2}\left|x_{i}(t)\right|^{p} \\
& +\sum_{i=1}^{n} \beta_{i}\left(\sum_{j=1}^{n}\left|a_{j i}\right| M_{j}\right)\left|x_{i}(t)\right|^{p} \\
& +(p-1) \sum_{i=1}^{n} M_{i}\left(\sum_{j=1}^{n}\left|b_{i j}\right| \beta_{j}\right)\left|x_{i}(t)\right|^{p} \\
& +\sum_{i=1}^{n} \beta_{i}\left(\sum_{j=1}^{n}\left|b_{j i}\right| M_{j}\right)\left|x_{i}(t-\tau)\right|^{p} \\
& (p-1)(p-2) \sum_{j=1}^{n} \sum_{i j}^{2}\left|x_{i}(t)\right|^{p} \\
&
\end{aligned}
$$




$$
\begin{aligned}
& +(p-1)(p-2) \sum_{i=1}^{n} \sum_{j=1}^{n} k_{i j}^{2}\left|x_{i}(t)\right|^{p} \\
& +2(p-1) \sum_{i=1}^{n} \sum_{j=1}^{n} k_{j i}^{2}\left|x_{i}(t-\tau)\right|^{p} .
\end{aligned}
$$

Taking the mathematical expectation on both sides of the above inequality and employing the expressions of $\lambda_{1}$ and $\lambda_{2}$, one has

$E \mathscr{L} V(x, t) \leqslant-\lambda_{1} E V(x(t), t)+\lambda_{2} E V(x(t-\tau), t-\tau)$.

Let $q \in\left(1, \lambda_{1} / \lambda_{2}\right), \lambda=\lambda_{1}-q \lambda_{2}$, then

$$
E V(s, x(s))<q E V(t, x(t)), \quad t-\tau \leqslant s \leqslant t
$$

which implies that

$$
E \mathscr{L} V(t, x(t)) \leqslant-\lambda E V(t, x(t)) .
$$

From the Lemma 2, we can conclude that

$$
E|x(t ; \xi)|^{p} \leqslant \frac{c_{2}}{c_{1}} E\|\xi\|^{p} e^{-\gamma t}, \quad \forall t \geqslant 0
$$

where $\gamma=\min \{\lambda, \log (q) / \tau\}$, which means that the trivial solution of system (1) is pth moment exponentially stable.

Corollary 4. Under Assumptions $(H 1)-(H 4)$, system (1) is exponentially stable in mean square if $\lambda_{1}>\lambda_{2}$, where

$$
\begin{aligned}
\lambda_{1}= & \min _{1 \leqslant i \leqslant n}\left\{2 \alpha_{i} m_{i}-M_{i}\left(\sum _ { j = 1 } ^ { n } \left(\left|a_{i j}\right|\right.\right.\right. \\
& \left.\left.\left.+\left|b_{i j}\right|\right) \beta_{j}\right)-\beta_{i} \sum_{j=1}^{n}\left|a_{j i}\right| M_{j}-2 \sum_{j=1}^{n} l_{j i}^{2}\right\}, \\
\lambda_{2}= & \max _{1 \leqslant i \leqslant n}\left\{\beta_{i} \sum_{j=1}^{n}\left|b_{j i}\right| M_{j}+2 \sum_{j=1}^{n} k_{j i}^{2}\right\} .
\end{aligned}
$$

Proof. By taking $p=2$ in Theorem 3, one can obtain the above result directly.

Theorem 5. Let Assumptions (H1) - (H4) hold, the trivial solution of system (1) is 1st moment exponentially stable, if

$$
\begin{array}{r}
-m_{i} \alpha_{i}+\beta_{i} \sum_{j=1}^{n} M_{j}\left(\left|a_{j i}\right|+\left|b_{j i}\right|\right)<0, \\
i=1,2, \ldots, n .
\end{array}
$$

Proof. Consider the following Lyapunov function

$$
V(x(t))=\sum_{i=1}^{n}\left|x_{i}(t)\right|
$$

By Itô's formula, the upper right Dini derivative $D^{+} V$ of $V$ along system (1) is

$$
\begin{aligned}
& D^{+}(V(x(t))) \\
= & \sum_{i=1}^{n} \operatorname{sign}\left(x_{i}(t)\right) d x_{i}(t) \\
\leqslant & \sum_{i=1}^{n}\left[-m_{i} \alpha_{i}\left|x_{i}(t)\right|+M_{i} \sum_{j=1}^{n}\left|a_{i j}\right| \beta_{j}\left|x_{j}(t)\right|\right. \\
& \left.+M_{i} \sum_{j=1}^{n}\left|b_{i j}\right| \beta_{j} \times\left|x_{j}(t-\tau)\right|\right] d t \\
& +\sum_{i=1}^{n} \sum_{j=1}^{n} \operatorname{sign}\left(x_{i}(t)\right) \sigma_{i j}\left(x_{j}(t), x_{j}(t-\tau)\right) d w_{j}(t) .
\end{aligned}
$$

Furthermore,

$$
\begin{aligned}
& D^{+}\left(e^{\gamma t} V(x(t))\right) \\
= & e^{\gamma t}\left(\gamma V(x(t)) d t+D^{+}(V(x(t)))\right) \\
\leqslant & e^{\gamma t}\left\{\sum _ { i = 1 } ^ { n } \left[\left(\gamma-m_{i} \alpha_{i}\right)\left|x_{i}(t)\right|+M_{i} \sum_{j=1}^{n}\left|a_{i j}\right| \beta_{j}\left|x_{j}(t)\right|\right.\right. \\
& \left.+M_{i} \sum_{j=1}^{n}\left|b_{i j}\right| \beta_{j}\left|x_{j}(t-\tau)\right|\right] d t \\
& +\sum_{i=1}^{n} \sum_{j=1}^{n} \operatorname{sign}\left(x_{i}(t)\right) \sigma_{i j}\left(x_{j}(t), x_{j}(t-\tau)\right) \\
& \left.\times d w_{j}(t)\right\} .
\end{aligned}
$$

Using the Itô's formula again, one can derive that

$$
\begin{aligned}
& e^{\gamma t} V(x(t)) \\
\leqslant & V(x(0))+\int_{0}^{t} e^{\gamma s}\left[\sum_{i=1}^{n}\left(\gamma-m_{i} \alpha_{i}\right)\left|x_{i}(s)\right|\right. \\
& +\sum_{i=1}^{n} \sum_{j=1}^{n} M_{i}\left|a_{i j}\right| \beta_{j}\left|x_{j}(s)\right|
\end{aligned}
$$




$$
\begin{aligned}
& \left.+\sum_{i=1}^{n} \sum_{j=1}^{n} M_{i}\left|b_{i j}\right| \beta_{j}\left|x_{j}(s-\tau)\right|\right] d s \\
& +\int_{0}^{t} \sum_{i=1}^{n} \sum_{j=1}^{n} e^{\gamma s} \operatorname{sign}\left(x_{i}(s)\right) \sigma_{i j}\left(x_{j}(s), x_{j}(s-\tau)\right) \\
& \times d w_{j}(s) \\
\leqslant & c+\int_{0}^{t} e^{\gamma s}\left[\sum _ { i = 1 } ^ { n } \left[\left(\gamma-m_{i} \alpha_{i}\right)\right.\right. \\
& \left.+\sum_{j=1}^{n} M_{j}\left|a_{j i}\right| \beta_{i}+e^{\gamma \tau} \sum_{j=1}^{n} M_{j}\left|b_{j i}\right| \beta_{i}\right] \times\left|x_{i}(s)\right| d s \\
& +\int_{0}^{t} \sum_{i=1}^{n} \sum_{j=1}^{n} e^{\gamma s} \operatorname{sign}\left(x_{i}(s)\right) \sigma_{i j}\left(x_{j}(s), x_{j}(s-\tau)\right) \\
& \times d w_{j}(s),
\end{aligned}
$$

here

$$
c=\sum_{i=1}^{n}\left|\varphi_{i}(0)\right|+e^{\gamma \tau} \sum_{i=1}^{n} \sum_{j=1}^{n} M_{i}\left|b_{i j}\right| \beta_{j} \int_{-\tau}^{0}\left|\varphi_{j}(s)\right| d s .
$$

From condition (8), one knows that for any $i(1 \leqslant$ $i \leqslant n)$, there exists a unique $\gamma_{i}$ such that

$$
\left(\gamma_{i}-m_{i} \alpha_{i}\right)+\sum_{j=1}^{n} M_{j}\left|a_{j i}\right| \beta_{i}+e^{\gamma_{i} \tau} \sum_{j=1}^{n} M_{j}\left|b_{j i}\right| \beta_{i}=0 .
$$

Let $\gamma=\min _{1 \leqslant i \leqslant n}\left\{\gamma_{i}\right\}$, then

$$
\begin{aligned}
& \left(\gamma-m_{i} \alpha_{i}\right)+\sum_{j=1}^{n} M_{j}\left|a_{j i}\right| \beta_{i}+e^{\gamma \tau} \sum_{j=1}^{n} M_{j}\left|b_{j i}\right| \beta_{i} \\
& \leqslant 0, \quad i=1,2, \ldots, n .
\end{aligned}
$$

Therefore, we can derive that

$$
\begin{aligned}
e^{\gamma t} V(x(t)) \leqslant & c+\int_{0}^{t} \sum_{i=1}^{n} \sum_{j=1}^{n} e^{\gamma s} \operatorname{sign}\left(x_{i}(s)\right) \\
& \times \sigma_{i j}\left(x_{j}(s), x_{j}(s-\tau)\right) d w_{j}(s) .
\end{aligned}
$$

Taking the mathematical expectation on both sides of the above inequality, one has

$$
e^{\gamma t} E V(x(t))=e^{\gamma t} E|x(t)|_{1} \leqslant c .
$$

Hence, we can conclude that

$$
E|x(t)|_{1} \leqslant c e^{-\gamma t},
$$

which means that the trivial solution of system (1) is $1 s t$ moment exponentially stable.

\section{Illustrative Example}

Consider the following delayed stochastic CGNN:

$$
\left\{\begin{aligned}
d x_{1}(t)= & -d_{1}\left(x_{1}\right)\left[c_{1}\left(x_{1}\right)-\sum_{j=1}^{2} a_{1 j} f_{j}\left(x_{j}(t)\right)\right. \\
& \left.-\sum_{j=1}^{2} b_{1 j} f_{j}\left(x_{j}(t-\tau)\right)\right] d t \\
& +\sum_{j=1}^{2} \sigma_{1 j}\left(x_{j}(t), x_{j}(t-\tau)\right) d w_{j}(t), \\
d x_{2}(t)= & -d_{2}\left(x_{1}\right)\left[c_{2}\left(x_{1}\right)-\sum_{j=1}^{2} a_{2 j} f_{j}\left(x_{j}(t)\right)\right. \\
& \left.-\sum_{j=1}^{2} b_{2 j} f_{j}\left(x_{j}(t-\tau)\right)\right] d t \\
& +\sum_{j=1}^{2} \sigma_{2 j}\left(x_{j}(t), x_{j}(t-\tau)\right) d w_{j}(t) ;
\end{aligned}\right.
$$

Let the functions $d_{1}\left(x_{1}\right)=5+\sin x, d_{2}\left(x_{2}\right)=4+$ $\cos x, c_{1}\left(x_{1}\right)=5 x_{1}, c_{2}\left(x_{2}\right)=7 x_{2}, f_{j}\left(x_{j}\right)=\frac{1}{2}\left(\mid x_{j}+\right.$ $\left.1|-| x_{j}-1 \mid\right), \sigma_{i j}\left(x_{j}, y_{j}\right)=x_{j}+y_{j}$, then one get $m_{1}=4, M_{1}=6, m_{2}=3, M_{2}=5, \alpha_{1}=8, \alpha_{2}=10$, $\beta_{1}=\beta_{2}=1, l_{i j}=k_{i j}=1$. Taking $a_{11}=a_{12}=a_{21}=$ $a_{22}=1, b_{11}=b_{12}=b_{21}=b_{22}=1$, we can see that the conditions of Corollary 1 hold. So the trivial solution of system (11) is exponentially stable in mean square.

In Wang et al's work ${ }^{11}$, the global asymptotic stability conditions for the neural network (1) can be written in the following form:

$$
\begin{aligned}
& \operatorname{diag}\left\{\alpha_{1}, \ldots, \alpha_{n}\right\}+M^{2}\left[\varepsilon_{1}^{-1} \lambda_{\max }\left(A A^{T}\right)\right. \\
& \left.+\varepsilon_{2}^{-1} \lambda_{\max }\left(B B^{T}\right)\right] I+\rho\left[\operatorname { d i a g } \left\{\sum_{j=1}^{n} l_{j 1}^{2},\right.\right. \\
& \left.\left.\ldots, \sum_{j=1}^{n} l_{j n}^{2}\right\}+\operatorname{diag}\left\{\sum_{j=1}^{n} k_{j 1}^{2}, \ldots, \sum_{j=1}^{n} k_{j n}^{2}\right\}\right] \\
& +\varepsilon_{1} \operatorname{diag}\left\{\beta_{1}^{2}, \ldots, \beta_{n}^{2}\right\}+\varepsilon_{2} \operatorname{diag}\left\{\beta_{1}^{2}, \ldots, \beta_{n}^{2}\right\} \\
& <0,
\end{aligned}
$$

where $m=\min _{1 \leqslant i \leqslant n}\left\{m_{i}\right\}, M=\max _{1 \leqslant i \leqslant n}\left\{M_{i}\right\}$ and $\rho>1$ is a constant. 
Taking the same parameters as above and calculating the left side of (12), we have

$$
\begin{aligned}
& -4 \times\left(\begin{array}{cc}
32 & 0 \\
0 & 31
\end{array}\right)+4^{2} \times 4 \times\left(\varepsilon_{1}^{-1}+\varepsilon_{2}^{-1}\right) I \\
& +\rho\left(\begin{array}{cc}
4 & 0 \\
0 & 4
\end{array}\right)+\left(\varepsilon_{1}+\varepsilon_{2}\right) \times\left(\begin{array}{cc}
16 & 0 \\
0 & 25
\end{array}\right) \\
\geqslant & \left(\begin{array}{cc}
4 & 0 \\
0 & 40
\end{array}\right) .
\end{aligned}
$$

One can see that the asymptotic stability condition of Wang et al ${ }^{11}$ is not satisfied, and the stability problem of system (11) can not be solved by Wang et al's criterion ${ }^{11}$. On the other hand, through a simple calculation one can see that the stability conditions of Theorem 3 is satisfied, and then system (11) is exponentially stable in the mean square by Theorem 3. Hence, the stability criteria provided here is more effective than the previous one.

Letting $d_{1}\left(x_{1}\right)=5+\sin x, d_{2}\left(x_{2}\right)=4+\cos x$, $c_{1}\left(x_{1}\right)=6 x_{1}, c_{2}\left(x_{2}\right)=8 x_{2}, f_{j}\left(x_{j}\right)=\frac{1}{2}\left(\left|x_{j}+1\right|-\right.$ $\left.\left|x_{j}-1\right|\right), \sigma_{i j}\left(x_{j}, y_{j}\right)=x_{j}+y_{j}$. one has $m_{1}=4, M_{1}=$ $6, m_{2}=3, M_{2}=5, \alpha_{1}=8, \alpha_{2}=10, \beta_{1}=\beta_{2}=1$, $l_{i j}=k_{i j}=1$. Further taking $a_{11}=a_{12}=a_{21}=a_{22}=$ $1, b_{11}=b_{12}=b_{21}=b_{22}=1$, it can be checked that the conditions of Theorem 5 is satisfied. So we can draw the conclusion that the trivial solution of system (11) is $1 s t$ moment exponentially stable.

\section{Conclusions}

In this paper, we have discussed the $\operatorname{pth}(p \geqslant 2)$ moment exponential stability and $1 s t$ moment exponential stability problem for the delayed stochastic CGNN. Instead of constructing Lyapunov functionals, we constructed some Lyapunov functions to derive the pth moment exponential stability criteria by using the stochastic version of Razumikhintype theorem which is more simple and can be easily checked. The 1 st moment exponential stability criteria is derived in a straightforward way and is also easy to be verified. A simple example has been used to demonstrate the usefulness of the obtained results. A comparison with the result given by Wang et al ${ }^{11}$ shows that our stability criterion is more effective than the existing one.

\section{Acknowledgments}

This work was supported by the National Natural Science Foundation of China under Grant No. 10801032 and the Teaching and Research Fund for Excellent Young Teachers at Southeast University of China.

\section{References}

1. M. Cohen and S. Grossberg, "Absolute stability and global pattern formation and parallel memory storage by competitive neural networks," IEEE Trans. on System, Man and Cybernetics, 13, 15-26(1983).

2. J. Cao and J. Liang, "Boundedness and stability for Cohen-Grossberg neural networks with time-varying delays," Journal of Mathematical Analysis and Applications, 296, 665-685(2004).

3. J. Cao and X. Li, "Stability in delayed CohenGrossberg neural network: LMI optimization approach," Physica D, 212, 54-65(2005).

4. X. Liao, C. Li and K. Wong, "Criteria for exponential stability of Cohen-Grossberg neural networks," Neural Networks, 17, 1401-1414(2004).

5. X. Liao and X. Mao, "Exponential stability and instability of stochastic neural network," Stochastic Analysis and Applications, 14, 165-185(1996).

6. X. Mao, Exponential Stability of Stochastic Differential Equations, Marcel Dekker, New York, 1994.

7. S. Blythe, X. Mao and X. Liao, "Stability of stochastic delay neural networks," Journal of the Franklin Institute, 338, 481-495(2001).

8. L. Wan and J. Sun, "Mean square exponential stability of stochastic delayed Hopfield neural networks," Physics Letter A, 343, 306-318(2005).

9. J. Hu, S. Zhong and L. Liang, "Exponential stability analysis of stochastic delayed cellular neural network," Chaos, Solitons \& Fractals, 27, 10061010(2006).

10. H. Zhao, and N. Ding, "Dynamic analysis of stochastic Cohen-Grossberg neural networks with time delays," Applied Mathematics and Computation, 183, 464-470(2006).

11. Z. Wang, Y. Liu, M. Li, and X. Liu, "Stability analysis for stochastic Cohen-Grossberg neural networks with mixed time delays," IEEE Trans. on Neural Networks, 17, 814-820(2006).

12. X. Mao, "Razumikhin-type theorems on exponential stability of stochastic tinctional differential equations," Stochastic Processes and their Applications, 65, 233-250(1996).

13. Q. Song, and Z. Wang, "Stability analysis of impulsive stochastic Cohen-Grossberg neural networks 
with mixed time delays," Physica A, 387, 33143326(2008).

14. Z. Wang, Y. Liu, K. Fraser, and X. Liu, "Stochastic stability of uncertain Hopfield neural networks with discrete and distributed delays," Physics Letters A ,354(4), 288-297(2006).

15. C. Yuan, and X. Mao, "Asymptotic stability in distribution of stochastic differential equations with Markovian switching," Stochastic Processes and their Applications, 103, 277-291(2003).

16. W. Yu, "Nonlinear system identifacation using discrete-time recurrent neural networks with stable learning algorithms," Information Sciences, 158, 131147(2004).

17. Y. He, G. Liu and D. Rees, "New delay-dependent stability criteria for neural networks with time-varying delay," IEEE Trans. on Neural Networks, 18(1), 310314(2007).
18. V. Kolmanovskii, and A. Myshkis, Introduction to the theory and applications of functional differential equations, Kluwer, Dordrecht, the Netherlands, 1999.

19. J. Liang, Z. Wang and X. Liu, "Exponential synchronization of stochastic delayed discrete-time complex networks," Nonlinear Dynamics, 53, 153-165(2008).

20. D. Yue and Q. Han, "Delay-dependent exponential stability of stochastic systems with time-varying delay, nonlinearity and Markovian switching," IEEE Trans. on Automatic Control, 50(2), 217-222(2005).

21. H. Gao, J. Lam and Z. Wang, "Discrete bilinear stochastic systems with time-varying delay: stability analysis and control synthesis," Chaos, Solitons \& Fractals, 34, 394-404(2007).

22. X. Mao, "Exponential stability in mean square of neutral stochastic differential functional equations," Systems \& Control Letters, 26, 245-251(1995). 